\title{
"Abusers" and "Addicts": Towards Abolishing Language of Criminality in US Medical Licensing Exam Step 1 Preparation Materials
}

\author{
Zoe M. Adams, BA, Elizabeth Fitzsousa, BA, and Marina Gaeta, BS@
}

Yale School of Medicine, New Haven, CT, USA.

J Gen Intern Med 36(6):1759-60

DOI: $10.1007 / \mathrm{s} 11606-021-06616-9$

() Society of General Internal Medicine 2021

$\mathrm{P}$ reparation materials for Step 1 of the United States Medical Licensing Exam (USMLE) describe patients with substance use disorders (SUDs) using outdated, stigmatizing terminology. In preparation for the Step 1 exam, students complete question banks with thousands of vignette-based, board-style questions and answer explanations. As medical students preparing for Step 1 in 2020, we noted terms like "abuser," "addict," and "alcoholic" within popular question banks (UWorld, Kaplan, and USMLERx) and National Board of Medical Examiners (NBME) practice exams. This language derives from the systematic criminalization of people who use drugs and has been replaced by contemporary terms (e.g., use disorders) within the medical community.

Terms like "substance abuser" perpetuate provider stigma and negatively influence patient care and outcomes. ${ }^{1}$ In 2013 , the Diagnostic and Statistical Manual of Mental Health Disorders (DSM-5) introduced contemporary diagnostic categories for SUDs and person-first terminology. Person-first terminology, originating from the disability rights movement, aims to humanize patients and retain their identities separate from their medical conditions. An example of person-first terminology is a "person with an opioid use disorder" as opposed to a "heroin user." Additionally, many medical fields have removed pejorative terms, like "abuser," "addict," or "alcoholic," from their literature. ${ }^{2}$ These changes aim to reduce the high level of bias healthcare providers harbor about SUDs, which discourages people with SUDs from seeking or continuing care and reduces the quality of care they receive. ${ }^{3}$

Step 1 is the first USMLE taken by aspiring physicians and integrates basic science into clinical scenarios. Students succeed by recognizing patterns and forming associations to identify

$\overline{\text { Zoe M. Adams, Elizabeth Fitzsousa and Marina Gaeta contributed equally }}$ to this work.

Received October 7, 2020

Accepted January 7, 2021

Published online January 28, 2021 medical conditions. On Step 1, a woman of childbearing age with dyspnea and a recent plane trip always has a pulmonary embolism; a patient who spelunks on weekends with a cough has histoplasmosis. In Step 1 preparation materials, patients with SUDs are not just mischaracterized as "addicts"; they are portrayed as irresponsible and negligent parents, "aggressive" and "uncooperative" patients, and "verbally abusive" to care providers. The 37-year-old who dies of pneumonia is called an "alcoholic" so students can easily identify Klebsiella; a cocaine "abuser" gets restrained in the Emergency Department for "belligerent" behavior so there's no question of his diagnosis; an IV drug "abuser" is "unwilling" to seek prenatal care and transmits HIV to her baby - cementing connections not just between HIV and IV drug use but neglectful parenting as well. Most students in the US sit for Step 1 before clinical rotations, making these patients in sample questions - depersonalized and without the opportunity to share their stories - their first exposure to patients with SUDs.

The terms "abuser" and "addict" stem from the historical framing of addiction as a moral failing. Colloquially, the word "abuse" is reserved for crimes by people with power exploiting those without, such as child abuse or sexual abuse. A highly effective rhetoric denouncing those who used substances as "drug abusers" in the 1960s and 70s reinforced associations between drug use and criminality. This fueled tough-on-crime federal policies, culminating in the War on Drugs. In the decades since, the average sentence length has nearly tripled and there are over ten times as many Americans incarcerated for drug-related charges. ${ }^{4}$ There is no evidence that criminalizing people who use drugs reduces substance use: data shows no correlation between imprisonment for drugs and drug use or overdose deaths. ${ }^{5}$

Prior to studying for Step 1, each of us already had personal and clinical experiences with people experiencing addiction. It was disturbing to realize medical students across the country were introduced to SUDs and people who have them in a very different way: harmful stereotypes and stigmatizing language. How could they not internalize this terminology when Step 1, by design, rewards pattern recognition that reinforces clinical and diagnostic stereotypes? We wondered whether question writers considered how a student with a personal or family experience of an SUD might feel reading these vignettes. Most importantly, we wondered how our patients might feel 
knowing that future physicians were purposefully taught to associate their brain disease with a moral failing - and then rewarded for perpetuating this harmful misconception.

This stigma against patients with SUDs strongly impacts their interactions with the medical system and tangibly worsens health experiences and outcomes. ${ }^{1,3,6}$ Thus, it was deeply troubling to encounter this language of criminality used to label patients with SUDs in USMLE preparation materials. These vignettes spanned all subject areas and body systems, and terms like "alcoholic" or "drug abuser" were included even when the patient's substance use was not relevant to the clinical scenario. We also noted a lack of contemporary language to describe SUDs. For example, the term "opioid use disorder" is absent from the 803-page First Aid for the USMLE Step 12020 edition, considered an essential preparatory resource. This book also incorrectly states that [opioid] "maintenance programs" are only "for heroin addicts" (page 551). Similarly, question bank vignettes about cirrhosis secondary to chronic alcohol use failed to mention "alcohol use disorder." Instead, questions called patients "alcoholics," saying "alcoholism" was a risk factor for their liver disease. This represents a missed opportunity to teach students person-first terminology and provide an accurate medical framework for addiction. If trainees are not taught correct terms, they may fall back on colloquial, stigmatizing language.

The persistence of inaccurate terminology cannot be explained by outdated materials. Question banks are continuously updated and review books release new editions annually. For example, the 2016 reclassification of Clostridium difficile to Clostridiodes was swiftly incorporated into study materials. If question bank and practice exam authors adopt other important language updates and frequently edit their work, why do vignettes regarding substance use and SUDs reflect medically inaccurate terminology 7 years after the DSM-5 was released?

Perhaps, this is a question of priority. Topics in addiction medicine require a deeper understanding of the social dimensions of illness and are historically undervalued in medical education. ${ }^{7}$ SUDs are rarely integrated into the 4 -year curriculum structure and SUD education materials are developed based on guidelines, not requirements. While individual programs have made strides in incorporating SUDs into their curricula, research on longitudinal interventions for teaching medical students about SUDs is limited; most refer to individual workshops rather than robust curricular changes, and few schools require a course in addiction medicine. ${ }^{7}$

Continued use of stigmatizing language produces and reinforces biases medical students will bring to encounters with real patients. Step 1 practice materials must be rewritten to remove stigmatizing language: we urge the NBME, UWorld, USMLERx, and other test preparation companies to consider the field of addiction medicine - and the lived experiences of its patients - as seriously as other disease processes. We encourage medical students, educators, and administrators to demand that test preparation companies update their materials using accurate terminology and information. We call on medical students to provide feedback to test prep companies about questions using stigmatizing language or negative stereotypes to describe patients with SUDs. Finally, we urge clinicians and medical educators to confront their biases and recognize the power their language has on the next generation of physicians.

Acknowledgements: We would like to acknowledge the support and encouragement of Dr. Anna Reisman and Dr. Declan Barry for their helpful input and guidance in writing this piece.

Corresponding Author: Marina Gaeta, BS; Yale School of Medicine, New Haven, CT 06511, USA (e-mail: Marina.gaeta@yale.edu).

\section{Compliance with Ethical Standards:}

Conflict of Interest: The authors declare that they do not have a conflict of interest.

\section{REFERENCES}

1. Kelly JF, Wakeman SE, Saitz R. Stop talking 'dirty': Clinicians, language, and quality of care for the leading cause of preventable death in the United States. Am J Med. 2015;128(1):8-9. https://doi.org/10.1016/j.amjmed. 2014.07.043.

2. Saitz R. Things that work, things that don't work, and things that matterincluding words. J Addict Med. 2015;9(6):429-30. https://doi.org/10. 1097/adm.0000000000000160.

3. van Boekel LC, Brouwers EPM, van Weeghel J, Garretsen HFL. Stigma among health professionals towards patients with substance use disorders and its consequences for healthcare delivery: Systematic review. Drug and Alcohol Dependence. 2013;131(1):23-35. https://doi.org/10.1016/j. drugalcdep.2013.02.018.

4. Fact Sheet: Trends in U.S. Corrections. Washington, D.C.: The Sentencing Project August 2020.

5. "More Imprisonment Does Not Reduce State Drug Problems" Pew Research Center, Washington, D.C. (2018). https://www.pewtrusts.org/en/research-and-analysis/issue-briefs/2018/03/more-imprisonment-doesnot-reduce-state-drug-problems.

6. Tsai AC, Kiang MV, Barnett ML, Beletsky L, Keyes KM, McGinty EE, et al. Stigma as a fundamental hindrance to the United States opioid overdose crisis response. PLOS Medicine. 2019;16(11):e1002969. https:// doi.org/10.1371/journal.pmed.1002969.

7. Muzyk A, Smothers ZPW, Akrobetu D, Ruiz Veve J, MacEachern M, Tetrault JM, et al. Substance use disorder education in medical schools: A scoping review. Academic Medicine. 2019;94(11):1825-34. https://doi. org/10.1097/acm.0000000000002883.

Publisher's Note: Springer Nature remains neutral with regard to jurisdictional claims in published maps and institutional affiliations. 